\title{
A priori estimates of the mass of the burnt materials in rooms of buildings in the integral mathematical model of the initial stage of the fire
}

\author{
Victoria Pavlidis, Yury Fedorov, Marina Chkalova, and Rayslu Suleymenova \\ Orenburg State Agrarian University, Institute of Risk Management and Complex Safety, \\ Chelyuskintsev str., 18, 460014 Orenburg, Russia
}

\begin{abstract}
The integral mathematical model of the initial stage of a fire in the premises of buildings focuses on the problem of estimating the mass of burnt materials assuming that the data on the rate of combustion of combustible materials are not complete. A priori estimates of the mass of burnt materials are found, which do not use data on the combustible material combustion rate. To derive these estimates, a special representation of the modulus of the increment (fall) of oxygen density in a room with a fire in the initial stage was preliminarily found. Two approaches to the derivation of a priori estimates of the mass of burnt materials have been implemented - using the upper and lower estimates of the modulus of the increment (fall) of the oxygen density. A comparison of a priori estimates obtained with different approaches is performed. Research can be useful at assessment of ecological situation and consequences of the fire.
\end{abstract}

\section{Introduction}

One of the main objectives of technical researches in agriculture is production risk mitigation.

The grain processing and grain-collecting facilities pose a significant threat for the population and environment. Modern technologies for receiving, storing and processing grain cause the accumulation of a significant amount of finely dispersed combustible organic materials at elevators, flour and feed mills. The explosive and fire-hazardous dust-air mixtures may occur in free spaces of technological, transport and aspiration equipment, as well as the production facilities and premises $[1,2]$. In addition, the fires and explosions at the grain storage and processing facilities can be caused by the self-heating process, which may develop into spontaneous combustion and malfunction of electrical equipment.

Therefore, fires at the grain processing plants pose a very serious danger, as they are often accompanied by explosions leading to death and injury of people, as well as to significant material damage. It is known that successful evacuation from the premises of public buildings in the event of a fire that is not accompanied by an explosion, as well as other actions to eliminate the danger to people in the event of a fire are possible only at the initial 
stage of fire development, until conditions incompatible with human life are reached [3, 4, 5].

The development of new, as well as the analysis and modernization of the existing mathematical models of fire (MMF) is a topical direction of modern engineering and applied research since the calculations of the dynamics of dangerous factors of fire (DFF) are the basic elements of fire risk (FR) assessment.

In the article under consideration, estimates of the mass of burnt materials are given for an integral mathematical model of the initial stage of a fire in the premises of buildings.

An integral mathematical model of a fire in the premises of buildings was developed by Yu.A. Koshmarov and his students [6, 7]. The integral mathematical model of the initial stage of a fire, which can be significantly simplified, corresponds to the generally accepted concept of a safe mass evacuation of people in the event of a fire in its initial stage $[5,6,7$, 8]. The theoretically possible assumptions for simplifying the model, as well as a simplified system of independent ordinary differential equations, to which the fire equations are reduced under such assumptions, were taken from [5] (equations (15) - (19) on page 407 of that paper). Earlier in $[9,10]$ for such a fire model, we found a priori estimates of the increments (falls) of such dangerous fire factors (DFF) as an increase in the concentration of toxic gases and a decrease in the oxygen concentration.

In this article, estimates of the mass of burnt materials are given for an integral mathematical model of the initial stage of a fire in the premises of buildings. To do this, in a simplified fire model, we have selected an equation for the average volumetric $r(t)$ oxygen density, $\mathrm{kg} / \mathrm{m}^{3}$

$$
V \frac{d r}{d t}=-h y(t)\left(k+k_{1} r\right)
$$

where $V$ is the volume of the room on fire $\left(\mathrm{m}^{3}\right) ; h$ - coefficient of completeness of combustion; function $y(t)$ - burnout rate $(\mathrm{kg} / \mathrm{s}) ; k$ - stoichiometric coefficient for oxygen (determines the amount of oxygen required for the combustion of a mass of combustible material), $\mathrm{kg} / \mathrm{kg} ; t$ - time (s);

$$
k_{1}=\frac{Q_{t}^{p}(1-j)+i_{\ni}}{c_{p} r_{0} T_{0}}
$$

[5] (equation (18), p.407). In formula (2) $Q_{t}^{p}$ - heat of combustion, $\mathrm{J} / \mathrm{kg} ; j$ - coefficient of heat loss; $i_{\ni}$ - enthalpy of gasification products of combustible material, $\mathrm{J} / \mathrm{kg} ; c_{p}-$ isobaric heat capacity of an ideal gas, $\mathrm{J} / \mathrm{kg} \mathrm{K} ; r_{0}, T_{0}$-the average volumetric density and temperature of the gaseous environment in the room before the start of the fire. The listed parameters at this stage of the fire are considered constant.

This model considers a continuous solution $r(t)$ to equation (1) with the initial condition

$$
r(0)=r_{0},(3)
$$

where $r_{0}$ is the oxygen density in the room before the start of the fire. After separating the variables in (1) and integrating by $s \mathrm{O}[0, t]$, we get: 


$$
\mathrm{T}_{0}^{t}\left(k+k_{1} r\right)^{-1} d r=-h V^{-1}{\underset{\mathrm{T}}{t}}_{0} y(s) d s .
$$

The solution $r(t)$ of the Cauchy problem (1), (3) can be represented by the exponent of function $M(t)$ on the right-hand side of (4)

$$
M(t)={\underset{0}{\mathrm{~T}}}_{0}^{t} y(s) d s,(5)
$$

expressing the law of change in the mass of combustible materials burnt by the time $t$.

If the speed of burning $y(t)$ is known, then $M(t)$ is found using the formula (5). Function $M(t)$ may have different appearance depending on conditions of burning and spreading mud process of the fire. For example, in [5], [7] the function $M(t)$ is presented in the form $M(t)=A t^{n}$, where $A=\frac{p}{3} y_{y d} v_{n}^{2}, n=3$ if the process of fire propagation over the surface of a solid combustible material ( $\mathrm{SCM}$ ) is circular; $A=\frac{b}{2} y_{y d} v_{l}, n=2$ if the spread of fire over the surface of the SCM is linear; $y_{y d}$ - specific mass rate of burnup $\left(\mathrm{kg} / \mathrm{m}^{2} \mathrm{~s}\right), v_{n}$ - linear speed of flame propagation $(\mathrm{m} / \mathrm{s}), b$ - width of the flame front $(\mathrm{m})$, and the flame propagates in one direction.

If the data on the burn-up rate $y(t)$ is incomplete, then the function $M(t)$ is unknown. Therefore, in this article, the following problem is considered: for the integral mathematical model of the initial stage of a fire in the premises of buildings, find a priori estimates of the mass of burnt materials $M(t)$ under conditions when the data on the burnout rate is $y(t)$ not complete, and all other necessary parameters of the model are known.

\section{Methods for a priori estimation of the mass of burnt materials with the incomplete data on the burn-up rate}

To estimate $M(t)$, we first find estimates $|\mathrm{D} r|$, where $\mathrm{D} r=r(t)-r_{0}$. We calculate the integral on the left-hand side of (4), then by potentiating we find

$$
\left(k+k_{1} r(t)\right)\left(k+k_{1} r_{0}\right)^{-1}=\exp \left(-k_{1} h V^{-1} M(t)\right) .
$$

We expand in Maclaurin's series the function $\exp \left(-k_{1} h V^{-1} M(t)\right)$ in (6)

$$
\exp \left(-k_{1} h V^{-1} M(t)\right)=\mathrm{e}_{m=0}^{\mathrm{r}}(-1)^{m} \frac{\left(k_{1} h V^{-1} M(t)\right)^{m}}{m !}
$$

which converges absolutely for all $k_{1} h V^{-1} M(t)$ i 0 [11] and transform the right-hand side of (7) 


$$
\exp \left(-k_{1} h V^{-1} M(t)\right)=1-k_{1} h V^{-1} M(t) \underset{j=0}{\mathrm{\Gamma}}(-1)^{j} \frac{\left(k_{1} h V^{-1} M(t)\right)^{j}}{(j+1) !} .
$$

Let us denote by $S(t)$ the sum of the series converging absolutely for the series $k_{1} h V^{-1} M(t)$ i 0

$$
S(t)=\mathrm{e}_{j=0}^{\mathrm{r}}(-1)^{j} \frac{\left(k_{1} h V^{-1} M(t)\right)^{j}}{(j+1) !} .
$$

Then formula (8) takes the form of

$$
\exp \left(-k_{1} h V^{-1} M(t)\right)=1-k_{1} h V^{-1} M(t) S(t) .
$$

Let us replace the right-hand side of equation (6) with the formula (10)

$$
\frac{k+k_{1} r(t)}{k+k_{1} r_{0}}=1-k_{1} h V^{-1} M(t) S(t)
$$

Finally, in (11), we transfer the unit to the left-hand side and simplify the resulting difference. As a result, we find a representation of the increment $\mathrm{D} r$ at the point $t=0$

$$
\begin{gathered}
\operatorname{Dr} \in r(t)-r_{0}=-b M(t) S(t), \\
b=h V^{-1}\left(k+k_{1} r_{0}\right) .
\end{gathered}
$$

It is more convenient to consider further $|\mathrm{D} r|$, since $\mathrm{D} r<0$ :

$$
|\mathrm{D} r|=b M(t)|S(t)|
$$

Sing-alternating series (9) converges pursuant to the Leibniz criterion for sufficiently small $M(t)$ (in a sufficiently small neighborhood of the point $t=0$ ), which are determined by the inequality

$$
k_{1} h V^{-1} M(t)<2 \text {, i.e., } M(t)<2 V\left(k_{1} h\right)^{-1}
$$

(the sequence of absolute values of the terms of series (9) is infinitesimal and decreases monotonically) and its sum $S(t)$ satisfies the estimate

$$
0<S_{2} \mathrm{~J} S(t) \mathrm{J} S_{1}
$$

where, $S_{1}=1, S_{2}=1-k_{1} h M(t)(2 V)^{-1}$ are the first and second partial sums of series (9) $[11]$.

Considering (14) and properties $S(t)(16)$, we conclude that

$$
|S(t)|=S(t),|\mathrm{D} r|=b M(t) S(t)
$$


From (16) and (17), we derive estimates of the modulus of the increment (fall) of oxygen density $|\mathrm{D} r|$ at the point $t=0$ in the integral mathematical model of the initial stage of the fire under the constraint (15)

$$
b M(t)\left(1-k_{1} h M(t)(2 V)^{-1}\right) \mathrm{J}|\mathrm{D} r| \mathrm{J} \quad b M(t) .
$$

Inequalities (18) allow to give estimates $M(t)$.

Pursuant to GOST 12.1.004-91, the normal oxygen content in the air is $0.226 \mathrm{~kg} / \mathrm{m} 3$, and the ratio $r_{0} / r_{0}=0,23$ [12]. A twofold decrease in this value leads to disturbances in the activity of the human pulmonary and cardiovascular systems, and the threefold decrease - to cessation of respiration and cardiac arrest [5]. It should be noted that with a drop in oxygen density $r(t)$, the modulus of increment (drop) of oxygen density $|\mathrm{D} r|$ increases. Therefore, we will consider the initial stage of the fire, at which the modulus of the increase (decrease) in the oxygen density does not exceed a certain critical value $|\mathrm{D} r| d_{\kappa p}$

$$
\begin{gathered}
|\mathrm{D} r| \mathrm{J} d_{k p}, \\
d_{k p}=|\mathrm{D} r|_{k p}=\left|a r_{0}-r_{0}\right|=(1-a) r_{0}, 0 \mathrm{~J} d_{k p} \mathrm{~J} r_{0},
\end{gathered}
$$

$a$ - the a priori selected coefficient that determines the critical value $d_{\kappa p}, 0<a<1$. For instance, if the density drop is allowed by a factor of two, then $r(t)$

$$
d_{k p}=r_{0} / 2
$$

\section{Analysis of two approaches to assessment of the burnt mate- rial mass}

Let us utilize and compare two approaches to estimation of $M(t)$, which are based on upper and lower estimates of $|\mathrm{D} r|$ in inequality (18). In (18), we first choose the right inequality, i.e., an upper $|\mathrm{D} r|$ bound, and adopt an additional, stronger than (19), constraint

$$
|\mathrm{D} r| \mathrm{J} b M(t) \mathrm{J} d_{\kappa p}
$$

Assessment $M(t)$ comes down to the solution of system linear inequalities (15) and (22)

$$
b M(t) \mathrm{J} d_{k p}, M(t)<2 V\left(k_{1} h\right)^{-1},
$$

Substituting instead of $b$ and $d_{\kappa p}$ their expressions from (13) and (20), respectively, we find the solution to the system of inequality (23) and, therefore, the estimate $M(t)$ in the first approach

$$
0 \mathrm{~J} M(t) \mathrm{J} V(1-a)\left(h\left(k+k_{1} r_{0}\right)\right)^{-1} r_{0} .
$$


In the second approach to the estimate $M(t)$, we compose a system of the left inequality (18) (lower $|\mathrm{D} r|$ bound) and constraint (15), assuming $|\mathrm{D} r| \mathrm{J} d_{k p}$ :

$$
b M(t)\left(1-k_{1} h M(t)(2 V)^{-1}\right) \mathrm{J} d_{k p}, M(t)<2 V\left(k_{1} h\right)^{-1}
$$

Having substituted in (25) instead of $b$ its expression from (13), we will have (25) as

$$
M^{2}(t)-\frac{2 V}{k_{1} h} M(t)+\left(\frac{2 V}{k_{1} h}\right)^{2} \frac{k_{1}}{2} \frac{d_{k p}}{\left(k+k_{1} r_{0}\right)} i 0, M(t)<2 V\left(k_{1} h\right)^{-1}
$$

Taking for the $d_{\kappa p}$ value (21) and finding the zeros $M_{1}$ and $M_{2}$ the quadratic function on the left-hand side of the first inequality in (26), we find the solution to the system of inequalities (26) as a set of the following inequalities

$$
0 \mathrm{~J} M(t)<M_{1}, M_{2}<M(t)<2 V\left(k_{1} h\right)^{-1} .
$$

where expressions for zero $M_{1}$ and $M_{2}$ have the appearance of

$$
M_{1}=V\left(k_{1} h\right)^{-1}\left(1-\sqrt{k\left(k+k_{1} r_{0}\right)^{-1}}\right), M_{2}=V\left(k_{1} h\right)^{-1}\left(1+\sqrt{k\left(k+k_{1} r_{0}\right)^{-1}}\right),
$$

and their borders, considering the inequality $0<k\left(k+k_{1} r_{0}\right)^{-1}<1$,

$$
0<M_{1}<V\left(k_{1} h\right)^{-1}, V\left(k_{1} h\right)^{-1}<M_{2}<2 V\left(k_{1} h\right)^{-1} .
$$

Based on the conditions of this model and the meaning of $M(t)$, we choose the first of inequalities (27) as an estimate of $M(t)$ in the second approach

$$
0 \mathrm{~J} M(t)<V\left(k_{1} h\right)^{-1}\left(1-\sqrt{k\left(k+k_{1} r_{0}\right)^{-1}}\right)
$$

Estimate (28) is more natural and accurate as compared to (24), since in deriving estimate (24) we used an additional artificial, stronger than (19), constraint (22).

\section{Conclusions}

1. In the integral mathematical model of the initial stage of a fire in the premises of buildings, the problem of estimating the mass of burnt materials assuming that the data on the rate of combustion of combustible materials are not complete is considered. A priori estimates (24) and (28) are found for the mass of burnt materials, which do not use data on the rate of combustion of combustible materials, and all other necessary parameters of the model are known.

2. To derive a priori estimates (24) and (28) of the mass of burnt materials, a special representation (17) was previously found for the modulus of increment (fall) of oxygen density in a room with a fire in the initial stage - a representation using the sum of an alternating series (9).

3. Two approaches have been implemented to derive a priori estimates of the mass of burnt materials based on estimate (18) from above and below the modulus of increment 
(fall) of oxygen density. A comparison of a priori estimates obtained with different approaches is performed.

4. Research can be useful at an assessment of ecological situation and consequences of the fires.

\section{References}

1. V.D. Pavlidis, Collection of scientific papers of the VII International Scientific and Practical Conference dedicated to the 60th anniversary of the creation of the first scientific unit in the Republic of Belarus in the field of prevention and elimination of emergencies and fires. In 2 parts, 245 (2016)

2. V.D. Pavlidis, Bulletin of Michurinsk branch of the Russian University of Cooperation, 4, 36 (2013)

3. M. Haghani, Safety Science, 128, 104745 (2020)

4. A. D’Orazio, L. Grossi, D. Ursetta, G. Carbotti, L. Poggi, International Journal of Safety and Security Engineering, 10(1), 1 (2020)

5. S.A. Kolodyazhny, I.I. Pereslavtseva, Bulletin of KGASU, Mathematical modeling, numerical methods and program complexes (in construction), 4 (30), 403 (2014)

6. Yu.A. Koshmarov, V.V. Rubtsov, Moscow Institute of Fire Safety of the Ministry of Internal Affairs of Russia, 90 (1998)

7. Yu.A. Koshmarov, Academy of State Fire Service of the Ministry of Internal Affairs of Russia, 118 (2000)

8. I.I. Pereslavtseva, S.A. Yaremenko, Bulletin of civil engineers, 5 (40), 122 (2013)

9. Yu.I. Fedorov, V.D. Pavlidis, V.A. Urban, Ye.V. Yakovleva, Safety in technosphere, 2 (83), 37 (2020)

10. Yu.I. Fedorov, V.D. Pavlidis, Scientific and technical bulletin of the Volga region, 2, $76(2021)$

11. L. D. Kudryavtsev, Mathematical Analysis, 1, 590 (1970)

12. GOST 30494-2011 Residential and public buildings 